\title{
Are we ready for genetic testing for primary open-angle glaucoma?
}

\author{
Anthony P. Khawaja $\mathbb{D}^{1} \cdot$ Ananth C. Viswanathan ${ }^{1}$
}

Received: 21 November 2017 / Revised: 14 December 2017 / Accepted: 16 December 2017 / Published online: 30 January 2018

(C) The Royal College of Ophthalmologists 2018

\begin{abstract}
Following a dramatic reduction in the cost of genotyping technology in recent years, there have been significant advances in the understanding of the genetic basis of glaucoma. Glaucoma patients represent around a quarter of all outpatient activity in the UK hospital eye service and are a huge burden for the National Health Service. A potential benefit of genetic testing is personalised glaucoma management, allowing direction of our limited healthcare resources to the glaucoma patients who most need it. Our review aims to summarise recent discoveries in the field of glaucoma genetics and to discuss their potential clinical utility. While genome-wide association studies have now identified over ten genes associated with primary openangle glaucoma (POAG), individually, variants in these genes are not predictive of POAG in populations. There are data suggesting some of these POAG variants are associated with conversion from ocular hypertension to POAG and visual field progression among POAG patients. However, these studies have not been replicated yet and such genetic testing is not currently justified in clinical care. In contrast, genetic testing for inherited early-onset disease in relatives of POAG patients with a known genetic mutation is of clear benefit; this can support either regular review to commence early treatment when the disease develops, or discharge from ophthalmology services of relatives who do not carry the mutation. Genetic testing for POAG at a population level is not currently justified.
\end{abstract}

\section{Introduction}

Glaucoma remains the second commonest cause of certifiable visual loss in England and Wales [1]. Given the chronic nature of glaucoma, lifelong follow-up is generally required. Therefore, glaucoma patients form a large proportion of outpatient activity in the UK hospital eye service (an estimated 23\% of all follow-up attendances) with over 1 million glaucoma-related visits per year [2, 3]. This represents a huge burden for the National Health Service (NHS) which is likely to grow further given the projected increase in the number of people with glaucoma [4]. Genetic testing offers the promise of personalised glaucoma management and directing limited healthcare resources to the patients that need it most.

There is strong evidence for a genetic contribution to the commonest form of glaucoma, primary open-angle

Anthony P. Khawaja

anthony.khawaja@ucl.ac.uk

$1 \quad$ NIHR Biomedical Research Centre, Moorfields Eye Hospital NHS Foundation Trust and UCL Institute of Ophthalmology, London, UK glaucoma (POAG). One twin study estimated the heritability of POAG to be $13 \%$, though this is a likely underestimate given glaucoma case ascertainment was via prescribing registries and that a considerable proportion of glaucoma in a population is undiagnosed [5]. First-degree relatives of POAG patients were shown to have a 9-fold increased risk of developing glaucoma in their lifetime compared to relatives of controls in the population-based Rotterdam Study [6]. Most convincingly, with the advent of affordable high-throughput DNA genotyping, there have now been multiple genes identified as contributing to susceptibility for POAG [7].

What is the future potential of genetic testing in the management of POAG? For patients already diagnosed with POAG, genetic testing may offer prognostic information, which may guide the intensity of their treatment and followup strategy. Genetic testing may also guide which treatments are most suitable for individual patients, predicting the most efficacious treatment and the treatment least likely to induce side effects. Within families with hereditary glaucoma, identifying the genetic cause will allow testing of offspring to determine who requires close monitoring and early treatment. The potential benefits are clear to see, but is our knowledge sufficient or our tools accurate and affordable enough that we are now ready for 
genetic testing in glaucoma management? Our review aims to answer these questions while giving a conceptual overview of POAG genetics and an update on recent advances in the field. The role of genetic testing in congenital glaucomas [8] is established and beyond the scope of this review.

\section{Search strategy}

We conducted the following Medline search: 'Genetic Testing' [Mesh] AND 'Glaucoma' [Mesh]. We further considered studies that were referenced in the articles identified in the initial search.

\section{Mendelian versus complex disease}

Mendelian disorders are conceptually the simplest demonstration of how genes can be responsible for disease. A single genetic defect alone causes a disease and if this is passed on by parents, their children will potentially inherit the disease. Common forms of inheritance of Mendelian disorders include autosomal dominant, autosomal recessive and X-linked recessive. If the genetic defect responsible for the disease in the family is identified, it is possible to screen offspring to determine their risk of disease and potentially take preventative action. For example, Angelina Jolie famously underwent bilateral mastectomy to prevent breast cancer knowing she had inherited the BRCAl gene mutation that had caused breast cancer in her family [9].

A complex disease is generally not caused by a single genetic defect; multiple genetic and/or environmental factors combine to collectively result in disease. Conceptually, it can be considered that each individual risk factor is insufficient to cause disease on its own and that each risk factor may not be present in all cases of disease (Fig. 1a). The fact that the risk factor may not be present in all cases and yet present in some controls makes identifying each individual risk factor challenging in complex disease. Large sample sizes are required to provide adequate power to identify each risk factor. An alternative way of conceptualising complex disease is shown in Fig. 1b-d. It may be that a single genetic factor is sufficient to cause disease, and that another single genetic factor is also sufficient to cause the disease, and these two different 'flavours' of the disease are indistinguishable or have not been separated during analyses. Again, in this situation also, important risk factors may not be present in all cases of the disease, posing a challenge for their identification. Similarly, large sample sizes can help identify each risk factor. Additionally, in this conceptual model, accurate phenotyping and separating (a)

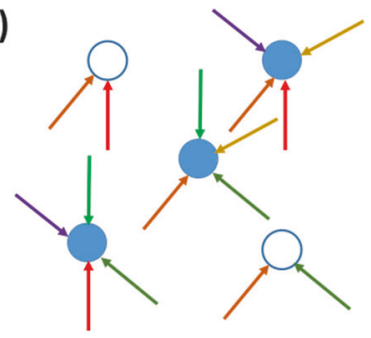

(c)

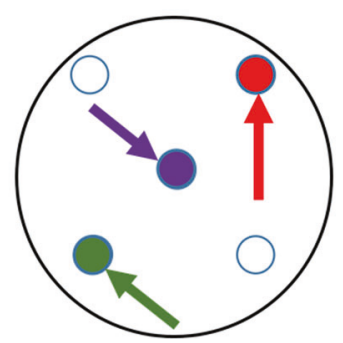

(b)

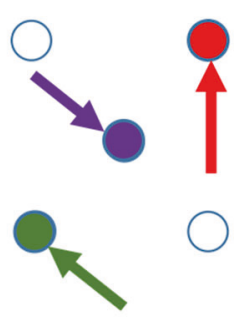

(d)

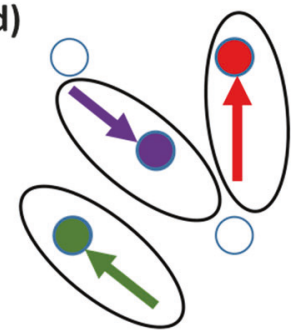

Fig. 1 Conceptual diagrams for complex disease. Each circle represents an individual person; filled in circles are people affected by disease and hollow circles are unaffected controls. $\mathbf{a}$ and $\mathbf{b}-\mathbf{d}$ are two different concepts for complex disease. First concept: a The arrows are risk factors (genetic or environmental); different colours represent different risk factors. It can be seen that none of the risk factors are present in all of the cases, and some of the risk factors that contribute to disease are present in controls. Second concept: b In this concept, each individual risk factor is sufficient to cause the disease. The different colours represent different subsets of disease which may or may not be clinically distinguishable. $\mathbf{c}$ If all cases are examined together, identifying each risk factor can be challenging as they are present only in subset of cases. d If the cases are subdivided in a biologically meaningful way, this can increase the power to identify risk factors despite the smaller sample size

cases into biologically meaningful subgroups can help improve power for detection of risk factors.

\section{Genetic mutations versus genetic variants}

As stated above, a Mendelian disease is caused by a single genetic alteration which is usually rare and is alone sufficient to cause a gene to malfunction and result in disease. Such genetic alterations are termed 'mutations'. 'Variants', on the other hand, are points in the genome (DNA code) at which we vary from one another. The human genome is $>3$ billion nucleotides long, but we vary at $<1 \%$ of these. The commonest form of variation is a nucleotide substitution at a single point in the genome and this is referred to as a single-nucleotide polymorphism (SNP). A genetic variant alone does not cause disease, but may be associated with disease. Possessing a variant may increase or decrease the risk of disease, but alone is insufficient to cause disease and is unlikely to be predictive of who will develop disease ( $c f$ arrows in Fig. 1a). Complex diseases may have many associated genetic variants. It is the cumulative contribution 
of these associations, or potentially interactions between them, that ultimately result in disease (Fig. 1a).

\section{Approaches to discovering genes that contribute to POAG}

Identifying a new gene for POAG in a hypothesisindependent manner requires methodology that looks for association across the whole genome. Until recently, it was not feasible to examine all independently inherited SNPs genome-wide. However, this was not necessary if examining genetic factors that segregate with disease in large families with inherited POAG. This approach is called linkage analysis and requires only around 400 markers to cover the whole genome. Linkage studies have identified several genes associated with glaucoma, such as myocilin (MYOC) [10], optineurin (OPTN) [11] and WD repeat domain 36 (WDR36) [12]. Mutations in these genes have been reported to cause autosomal dominant Mendelian POAG in the studied families. Further details on the roles of these genes in POAG have been previously reviewed [7, 13, 14]. While a mutation in one of these genes may completely explain the development of POAG in some families, collectively, mutations in these genes contribute to only around $6 \%$ of POAG cases in the general population [15-17]. More recently, family studies have identified TANK binding kinase $1(T B K 1)$ as another cause of Mendelian POAG [18]. Rather than a mutation within the gene, it is duplication of the gene and the resultant increase in function that appears to be causing the glaucomatous process.

The cost of genome-wide genotyping has fallen dramatically in recent years, at a rate much faster than Moore's Law. This has resulted in affordable highthroughput technologies that can measure all common independently inherited genetic variation across the whole genome in individuals. Therefore, it has become possible to investigate genetic associations with POAG, hypothesis independent and genome-wide, without the need for families. Instead, unrelated POAG cases are collected and compared with unrelated controls at several million genetic markers (some directly measured and others imputed based on reference data). This approach is called a genome-wide association study (GWAS). GWAS identifies common variants (with a frequency of over $5 \%$ in the general population) associated with disease. Given the number of genetic variants examined, there is a multiple testing statistical issue. For this reason, associations are only considered significant and valid if the $P$-value is very small (a 'hit' is considered to be 'genome-wide significant' if $P<5 \times 10^{-8}$ ) and there is evidence for the same association in an independent cohort. The first glaucoma GWAS discovery was the LOXL1 locus for exfoliation glaucoma [19]. The first replicated GWAS discovery for POAG was in an Icelandic population which identified a significant locus near CAVI and CAV2 (both of which are expressed in retinal ganglion cells and trabecular meshwork) [20]. Further GWAS of European populations have identified other significant POAG loci in discovery cohorts from the United States [21, 22] (near or at SIX1/SIX6, TXNRD2, ATXN2 and FOXC1) and Australia [23, 24] (TMCO1, CDKN2B-AS1, ABCA1, $A F A P 1$ and $G M D S)$. A POAG GWAS in people of Chinese descent identified a significant locus in PMM2 [25]. Despite these identified variants being common, the effect of each one is small, and collectively they explain only a small fraction $(<5 \%)$ of POAG heritability. It is anticipated many more loci will be identified as the statistical power improves with a larger sample of POAG cases. Following the initial LOXL1 locus discovery, the combination of cases from a large international consortium has identified further exfoliation glaucoma loci at CACNA1A [26], POMP, TMEM136, AGPAT1, RBMS3 and SEMA6A [27]. There has also been some GWAS success for primary angle-closure glaucoma, with eight genetic loci identified to date (near or at PLEKHA7, COL11A1, EPDR1, PCMTD1-ST18EPDR1, CHAT, GLIS3, FERMT2 and DPM2-FAM102A) [28, 29].

There have also been multiple GWAS hits for heritable quantitative traits related to glaucoma (endophenotypes), such as intraocular pressure (IOP), and optic cup-disc ratio (CDR). A large IOP GWAS from the International Glaucoma Genetics Consortium (IGGC) identified GAS7 as a significant locus for both IOP and POAG [30]. There have been over 40 genetic loci identified for CDR in the largest published GWAS meta-analysis from the IGGC [31]. However, it remains unclear what role these loci have in disease, as the majority do not demonstrate association with POAG when tested in the available cohorts. It is possible these variants are related to developmental processes and associated with optic disc anatomy and not the pathological glaucomatous cupping process. Alternatively, these variants are associated with POAG aetiological processes, but there is insufficient power in the currently available POAG case-control datasets to confirm the associations.

\section{Evidence for clinical utility of genetic testing in POAG}

Learning which genes contribute to POAG can inform us about previously unknown biological pathways that are important in disease aetiology and progression. In the longer term, these discoveries can prompt further research into these pathways and potentially lead to new treatments. 
In the shorter term, it is possible that genetic markers are of predictive value and can help personalise glaucoma management.

\section{Diagnosis in hereditary POAG}

There are situations when genetic testing can be helpful for managing families with inherited POAG. For example, a young member of a family with severe, early onset, autosomal dominant POAG may benefit from knowing their likelihood of developing the disease [32]. If the mutation causing POAG in that family is identified (e.g., by testing for myocilin mutations in affected family members), then the individual concerned can be tested for that mutation. If they do not carry the myocilin mutation, then their risk of developing POAG will be similar to the risk in the general population, and this would allow discharge from routine ophthalmic examinations [32]. Such information may even inform life choices such as occupation, especially if the disease is of early onset. Conversely, if they do carry the mutation, this would warrant regular follow-up for early signs of raised IOP and permit early treatment.

More general screening of relatives for an identified disease-causing mutation is termed cascade genetic testing. There is some evidence that early diagnosis and treatment of myocilin-related POAG following cascade genetic testing may result in a better clinical outcome. In a retrospective study, glaucoma severity parameters were compared between patients who were identified by cascade genetic testing (Genetic cases) and patients who presented through normal clinical pathways and were subsequently found to have a myocilin mutation (Clinical cases) [33]. Clinical cases had significantly higher maximum IOP, larger CDR and worse visual field mean deviation than Genetic cases [33].

It has been suggested there may be benefit in screening patients with advanced POAG for myocilin mutations if they meet certain criteria (young age of onset, high maximum IOP and strong family history) [34]. The prevalence of myocilin mutations in this phenotypically selected group ranged from $16 \%$ to $40 \%$ depending on the cutoff thresholds. Identification of a myocilin mutation could then prompt cascade genetic testing and early treatment of family members at high risk [34].

Deciding whether to test patients or family members for myocilin mutations may not be straight-forward and genetic counselling should be offered [35]. This may involve referral to a clinical genetics service. Information provided should include details about the condition and its prognosis, its inheritance pattern, and risk to children or other family members. Counselling for at-risk but currently unaffected family members should explore the underlying motivation for genetic testing, and explain the testing process and potential impact of the test result [35]. Accredited testing for myocilin mutations is currently available to NHS clinicians via the UK Genetic Testing Network [36]. At the time of writing, sequencing the entire myocilin gene to look for any mutation cost $£ 305$, whereas testing for one known mutation in a family member cost $£ 180$ [36]. There is currently regional variation on whether commissioners will cover the cost of myocilin genetic testing.

\section{Predicting conversion from ocular hypertension (OHT) to POAG}

A subset of participants of the Ocular Hypertension Treatment Study (OHTS) were genotyped for variants previously associated with POAG and these variants tested for association with subsequent conversion from OHT to POAG [37]. Among the largest ethnic group in cohort, nonHispanic Whites, a SNP in TMCO1 was significantly associated with the development of POAG. TMCO1 has been strongly associated with IOP [30, 38] and it is assumed that the variant mediates its increased risk of POAG by raised IOP. Remarkably, the association between the TMCOI variant and POAG conversion remained highly significant even after adjustment for all parameters in the previously published risk calculator [39], including baseline IOP; the hazard ratio was 1.7 per risk allele $(95 \%$ confidence interval $1.3-2.3, P=0.0004$ ) [37]. This equates to a 3 -fold increased risk of POAG in people with two risk alleles compared to people with no risk alleles, an effect size that is comparable to other established risk factors such as age. It is perhaps surprising that the TMCOI effect remains significant even after adjustment for a direct measurement of IOP. This suggests that the TMCOI variant provides information regarding the cumulative level of true IOP over and above that provided by a single measurement at baseline. While this is an exciting finding that offers hope for the potential of genetic testing in the management of OHT, replication of this finding in an independent study would provide stronger evidence. It should also be noted that there was no discernible association between the TMCOI variant and conversion to POAG in the Black subgroup [37].

\section{Predicting progression of POAG}

Examining risk factors for susceptibility to progression of POAG in treated cohorts is challenging, not least because intensity of treatment is difficult to quantify and account for. A study of 469 Singaporean Chinese POAG patients with 5 or more visual fields showed that only one of ten POAG loci tested was associated with visual field progression 
(ascertained by pointwise linear regression criteria) [40]. This locus was in the TGFBR3-CDC7 region and was associated with a 6.7 (95\% CI 1.9-23.7, $P=0.003)$ times increased chance of visual field progression. The wide confidence interval suggests uncertainty of this effect estimate and there is a possibility this is a chance finding. Replication in an independent cohort is required before firm conclusions can be made. Unfortunately, data for the TMCOI variant that was examined in OHTS were not available for this study.

\section{Predicting response to treatment}

There is good evidence that, in general, there may be a genetic basis for effectiveness of treatment in different individuals, as well as for the development of side effects for treatment [41]. However, pharmacogenomic studies for glaucoma treatments have been small and with conflicting results. For example, variants in the prostaglandin $F 2 \alpha$ gene have been associated with response to prostaglandin analogues in Japanese studies [42, 43] but not in a North American study [44]. A variant in ADRB2 has been associated with response to timolol drops, but this finding remains unreplicated [45]. Currently, there is no convincing evidence for genetic testing to support the choice of treatment for POAG.

\section{Targeted therapy for Mendelian POAG}

Identifying the disease-causing mutation in Mendelian POAG offers the potential for targeted therapy to fix the specific molecular defect caused by the mutation. It has been suggested that myocilin mutations result in misfolded MYOC protein accumulating in trabecular meshwork cells resulting in an adverse effect [46]. Phenylbutyrate, a chemical chaperone that aids proteins folding into their correct conformations, appears to cure myocilin-caused glaucoma in transgenic mice when administered orally or as an eyedrop [46, 47]. While phenylbutyrate has not been tested in humans, this may serve as a proof of concept for targeting treatment to the underlying pathology caused by a specific genetic defect.

More recently, clustered regularly interspaced short palindromic repeats (CRISPR)-mediated genome editing was used to disrupt the mutant myocilin gene in a mouse model, resulting in reduced endoplasmic reticulum stress, lower IOP, and prevention of further glaucomatous damage [48]. Additionally, the investigators demonstrated the potential feasibility of human genome editing in the eye using an ex vivo human organ culture system [48].

\section{Conclusions}

The pace of new genetic discoveries for glaucoma has increased significantly in recent years due to the exponential drop in cost of high-throughput genome-wide genotyping platforms. While there is some evidence supporting the clinical utility of this new knowledge, such as TMCO1 variation being predictive of conversion from OHT to POAG, such studies are small and not replicated to date. Genetic testing for glaucoma is clearly helpful in some specific situations, such as screening of family members in autosomal dominant POAG of early onset. POAG pharmacogenomics is an understudied area that warrants further work in the GWAS-era. However, genetic testing for POAG at a population level is not currently justified. We look forward to further genetic discoveries for glaucoma as statistical power increases, from large cohorts such as the UK Biobank and from global collaborations such as the IGGC. Time will tell if these discoveries will help us manage our patients better, or at least help direct resources to those who need them most.

Acknowledgements We thank Professor Andrew Lotery for his advice on $M Y O C$ testing in the UK. Anthony Khawaja is supported by a Moorfields Eye Charity grant.

\section{Compliance with ethical standards}

Conflict of interest The authors declare that they have no competing interests.

\section{References}

1. Quartilho A, Simkiss P, Zekite A, Xing W, Wormald R, Bunce C. Leading causes of certifiable visual loss in England and Wales during the year ending 31 March 2013. Eye (Lond). 2016;30 (4):602-7.

2. Spry PG, Spencer IC, Sparrow JM, Peters TJ, Brookes ST, Gray S, et al. The Bristol Shared Care Glaucoma Study: reliability of community optometric and hospital eye service test measures. Br J Ophthalmol. 1999;83(6):707-12.

3. King A, Azuara-Blanco A, Tuulonen A. Glaucoma. BMJ. 2013;346(June):f3518.

4. Tham Y-C, Li X, Wong TY, Quigley HA, Aung T, Cheng C-Y. Global prevalence of glaucoma and projections of glaucoma burden through 2040: a systematic review and meta-analysis. Ophthalmology. 2014;121(11):2081-90.

5. Teikari JM. Genetic factors in open-angle (simple and capsular) glaucoma. A population-based twin study. Acta Ophthalmol. 1987;65(6):715-20.

6. Wolfs RC, Klaver CC, Ramrattan RS, van Duijn CM, Hofman A, de Jong PT. Genetic risk of primary open-angle glaucoma. Population-based familial aggregation study. Arch Ophthalmol. 1998;116(12):1640-5.

7. Liu Y, Allingham RR. Major review: molecular genetics of primary open-angle glaucoma. Exp Eye Res. 2017;160: $62-84$. 
8. Lewis CJ, Hedberg-Buenz A, DeLuca AP, Stone EM, Alward WLM, Fingert JH. Primary congenital and developmental glaucomas. Hum Mol Genet. 2017;26(R1):R28-R36.

9. Ludwig KK, Neuner J, Butler A, Geurts JL, Kong AL. Risk reduction and survival benefit of prophylactic surgery in BRCA mutation carriers, a systematic review. Am J Surg. 2016;212(4):660-669.

10. Morissette J, Côté G, Anctil JL, Plante M, Amyot M, Héon E, et al. A common gene for juvenile and adult-onset primary openangle glaucomas confined on chromosome 1q. Am J Hum Genet. 1995;56(6):1431-42.

11. Rezaie T, Child A, Hitchings R, Brice G, Miller L, Coca-Prados $\mathrm{M}$, et al. Adult-onset primary open-angle glaucoma caused by mutations in optineurin. Science. 2002;295(5557):1077-9.

12. Monemi S, Spaeth G, DaSilva A, Popinchalk S, Ilitchev E, Liebmann $\mathrm{J}$, et al. Identification of a novel adult-onset primary open-angle glaucoma (POAG) gene on 5q22.1. Hum Mol Genet. 2005; 14(6):725-33.

13. Miller MA, Fingert JH, Bettis DI. Genetics and genetic testing for glaucoma. Curr Opin Ophthalmol. 2016;28(2):133-38.

14. Gemenetzi M, Yang Y, Lotery AJ. Current concepts on primary open-angle glaucoma genetics: a contribution to disease pathophysiology and future treatment. Eye (Lond). 2012;26(3):355-69.

15. Fingert JH, Héon E, Liebmann JM, Yamamoto T, Craig JE, Rait J, et al. Analysis of myocilin mutations in 1703 glaucoma patients from five different populations. Hum Mol Genet. 1999;8 (5):899-905.

16. Alward WL, Kwon YH, Kawase K, Craig JE, Hayreh SS, Johnson AT, et al. Evaluation of optineurin sequence variations in 1,048 patients with open-angle glaucoma. Am J Ophthalmol. 2003;136 (5):904-910.

17. Hauser MA, Allingham RR, Linkroum K, Wang J, LaRocqueAbramson K, Figueiredo D, et al. Distribution of WDR36 DNA sequence variants in patients with primary open-angle glaucoma. Invest Ophthalmol Vis Sci. 2006;47(6):2542-6.

18. Fingert JH, Robin AL, Stone JL, Roos BR, Davis LK, Scheetz TE, et al. Copy number variations on chromosome 12q14 in patients with normal tension glaucoma. Hum Mol Genet. 2011;20 (12):2482-2494.

19. Thorleifsson G, Magnusson KP, Sulem P, Walters GB, Gudbjartsson DF, Stefansson $\mathrm{H}$, et al. Common sequence variants in the LOXL1 gene confer susceptibility to exfoliation glaucoma. Science. 2007;317(5843):1397-400.

20. Thorleifsson G, Walters GB, Hewitt AW, Masson G, Helgason A, DeWan A, et al. Common variants near CAV1 and CAV2 are associated with primary open-angle glaucoma. Nat Genet. 2010;42(10):906-9.

21. Wiggs JL, Yaspan BL, Hauser MA, Kang JH, Allingham RR, Olson LM, et al. Common variants at 9p21 and 8q22 are associated with increased susceptibility to optic nerve degeneration in glaucoma. PLoS Genet. 2012;8(4):e1002654.

22. Bailey JNC, Loomis SJ, Kang JH, Allingham RR, Gharahkhani P, Khor CC, et al. Genome-wide association analysis identifies TXNRD2, ATXN2 and FOXC1 as susceptibility loci for primary open-angle glaucoma. Nat Genet. 2016;48(2):189-94.

23. Burdon KP, Macgregor S, Hewitt AW, Sharma S, Chidlow G, Mills RA, et al. Genome-wide association study identifies susceptibility loci for open angle glaucoma at TMCO1 and CDKN2B-AS1. Nat Genet. 2011;43(6):574-8.

24. Gharahkhani P, Burdon KP, Fogarty R, Sharma S, Hewitt AW, Martin S, et al. Common variants near ABCA1, AFAP1 and GMDS confer risk of primary open-angle glaucoma. Nat Genet. 2014;46(10):1120-1125.

25. Chen Y, Lin Y, Vithana EN, Jia L, Zuo X, Wong TY, et al. Common variants near ABCA1 and in PMM2 are associated with primary open-angle glaucoma. Nat Genet. 2014;46(10): 1115-1119.
26. Aung T, Ozaki M, Mizoguchi T, Allingham RR, Li Z, Haripriya $\mathrm{A}$, et al. A common variant mapping to CACNA1A is associated with susceptibility to exfoliation syndrome. Nat Genet. 2015;47 (4):387-392.

27. Aung T, Ozaki M, Lee MC, Schlötzer-Schrehardt U, Thorleifsson G, Mizoguchi T, et al. Genetic association study of exfoliation syndrome identifies a protective rare variant at LOXL1 and five new susceptibility loci. Nat Genet. 2017;49(7):993-1004.

28. Khor CC, Do T, Jia H, Nakano M, George R, Abu-Amero K, et al. Genome-wide association study identifies five new susceptibility loci for primary angle closure glaucoma. Nat Genet. 2016;48 (5):556-562.

29. Vithana EN, Khor C-C, Qiao C, Nongpiur ME, George R, Chen L-J, et al. Genome-wide association analyses identify three new susceptibility loci for primary angle closure glaucoma. Nat Genet. 2012;44(10):1142-6.

30. Hysi PG, Cheng C-Y, Springelkamp H, Macgregor S, Bailey JNC, Wojciechowski R, et al. Genome-wide analysis of multi-ancestry cohorts identifies new loci influencing intraocular pressure and susceptibility to glaucoma. Nat Genet. 2014;46(10):1126-30.

31. Springelkamp H, Iglesias AI, Mishra A, Höhn R, Wojciechowski $\mathrm{R}$, Khawaja AP, et al. New insights into the genetics of primary open-angle glaucoma based on meta-analyses of intraocular pressure and optic disc characteristics. Hum Mol Genet. 2017;26 (2):438-453.

32. Souzeau E, Glading J, Ridge B, Wechsler D, Chehade M, Dubowsky A, et al. Predictive genetic testing in minors for Myocilin juvenile onset open angle glaucoma. Clin Genet. 2015;88(6):584-588.

33. Souzeau E, Tram KH, Witney M, Ruddle JB, Graham SL, Healey $\mathrm{PR}$, et al. Myocilin Predictive Genetic Testing for Primary OpenAngle Glaucoma Leads to Early Identification of At-Risk Individuals. Ophthalmology. 2017;124(3):303-309.

34. Souzeau E, Burdon KP, Dubowsky A, Grist S, Usher B, Fitzgerald JT, et al. Higher prevalence of myocilin mutations in advanced glaucoma in comparison with less advanced disease in an australasian disease registry. Ophthalmology. 2013;120 (6): $1135-43$.

35. Gillespie RL, Hall G, Black GC. Genetic testing for inherited ocular disease: delivering on the promise at last? Clin Exp Ophthalmol. 2014;42(1):65-77.

36. UK Genetic Testing Network. Myocilin genetic testing. Available at: https://ukgtn.nhs.uk/find-a-test/search-by-disorder-gene/gla ucoma-1-open-angle-a-178/ [Accessed December 14, 2017].

37. Scheetz TE, Faga B, Ortega L, Roos BR, Gordon MO, Kass MA, et al. Glaucoma risk alleles in the ocular hypertension treatment study. Ophthalmology. 2016;123(12):2527-2536.

38. van Koolwijk LME, Ramdas WD, Ikram MK, Jansonius NM, Pasutto F, Hysi PG, et al. Common genetic determinants of intraocular pressure and primary open-angle glaucoma. PLoS Genet. 2012;8(5):e1002611.

39. Gordon MO, Torri V, Miglior S, Beiser JA, Floriani I, Miller JP, et al. Validated prediction model for the development of primary open-angle glaucoma in individuals with ocular hypertension. Ophthalmology. 2007;114(1):10-9.

40. Trikha S, Saffari E, Nongpiur M, Baskaran M, Ho H, Li Z, et al. A genetic variant in TGFBR3-CDC7 is associated with visual field progression in primary open-angle glaucoma patients from Singapore. Ophthalmology. 2015;122(12):2416-2422.

41. Whirl-Carrillo M, McDonagh EM, Hebert JM, Gong L, Sangkuhl $\mathrm{K}$, Thorn CF, et al. Pharmacogenomics Knowledge for Personalized Medicine. Clin Pharmacol Ther. 2012;92(4):414-417.

42. Sakurai M, Higashide T, Takahashi M, Sugiyama K. Association between genetic polymorphisms of the prostaglandin $\mathrm{F} 2 \alpha$ receptor gene and response to latanoprost. Ophthalmology. 2007;114 (6):1039-1045. 
43. Sakurai M, Higashide T, Ohkubo S, Takeda H, Sugiyama K. Association between genetic polymorphisms of the prostaglandin F2 $\alpha$ receptor gene, and response to latanoprost in patients with glaucoma and ocular hypertension. Br J Ophthalmol. 2014;98 (4):469-473.

44. McCarty CA, Berg R, Patchett R, Wilke RA, Burmester JK. Lack of association between polymorphisms in the prostaglandin $\mathrm{F} 2 \alpha$ receptor and solute carrier organic anion transporter family $2 \mathrm{~A} 1$ genes and intraocular pressure response to prostaglandin analogs. Ophthalmic Genet. 2012;33(2):74-6.

45. McCarty CA, Burmester JK, Mukesh BN, Patchett RB, Wilke RA. Intraocular pressure response to topical $\beta$-blockers associated with an ADRB2 single-nucleotide polymorphism. Arch Ophthalmol. 2008;126(7):959.
46. Zode GS, Kuehn MH, Nishimura DY, Searby CC, Mohan K, Grozdanic SD, et al. Reduction of ER stress via a chemical chaperone prevents disease phenotypes in a mouse model of primary open angle glaucoma. J Clin Invest. 2011;121 (9):3542-53.

47. Zode GS, Bugge KE, Mohan K, Grozdanic SD, Peters JC, Koehn DR, et al. Topical ocular sodium 4-phenylbutyrate rescues glaucoma in a myocilin mouse model of primary openangle glaucoma. Investig Ophthalmol Vis Sci. 2012;53(3): $1557-1565$.

48. Jain A, Zode G, Kasetti RB, Ran FA, Yan W, Sharma TP, et al. CRISPR-Cas9-based treatment of myocilin-associated glaucoma. Proc Natl Acad Sci. 2017;114(42):11199-204. (32): 201706193. 